\title{
Method for Screening Ecdysone-Inducible Stable Cell Lines
}

BioTechniques 31:414-418(August 2001)

\author{
Kenichi Wakita ${ }^{1,2}$, Frank \\ McCormick ${ }^{1}$, and Osamu \\ Tetsu $^{1}$ \\ 'University of California, San \\ Francisco, San Francisco, CA, \\ USA, and ${ }^{2}$ Daiichi Pharmaceuti- \\ cal Co. Ltd., Tokyo, Japan
}

\begin{abstract}
Ecdysone-inducible systems are useful tools to study the function of various genes in different types of mammalian cells. However, it is technically difficult to establish stable cell lines. This is partly because the conditional expression system requires the expression of two or more components driven by different kinds of promoters. In this paper, we describe the use of a luciferase reporter gene system for rapid screening of cell lines that express the ecdysone and retinoid $X$ receptors. Using this system, two human stable colon cancer cell lines, SW480/VgRXR and $H C T 116 / \mathrm{VgRXR}$, were successfully generated. The expression of these receptors remained high after six months of continuous culturing. A tight regulation of gene induction in SW480/VgRXR was observed using 2 $\mu M$ Ponasterone A. The gene induction was rapid and persistent. Our results demonstrated the advantage of establishing cell lines that continuously express high levels of ecdysone receptor proteins.
\end{abstract}

\section{INTRODUCTION}

The ecdysone-inducible system is one of several commercially available exogenous gene-inducible systems for mammalian cells (Invitrogen, Carlsbad, CA, USA). In the presence of ecdysone or its analog, heterodimeric retinoid $\mathrm{X}$ receptor (RXR) and ecdysone receptor (VgEcR), both encoded in the $\mathrm{pVgRXR}$ vector, form a complex and bind to the ecdysone response element in the pIND expression vector. Expression of the target gene is thereby transactivated.

Despite the well-designed mechanism, there remain technical difficulties in the process of establishing stable ecdysone-inducible cell lines such as leakage of target gene expression and/or low or no induction of gene expression. The latter can be due to low expression of either the receptors or the transgene. It is difficult to determine which factor is responsible for low expression if all components are transfected simultaneously. To more efficiently generate cell lines, it is advantageous to first establish a cell line that stably expresses the receptor proteins RXR and $\mathrm{VgEcR}$ proteins. However, so far, no rapid, sensitive, and quantitative screening system is available.

To facilitate the identification of cells that functionally express the ecdysone receptor, Invitrogen supplies a lacZ-based reporter system, pIND/ lac $Z$, that exhibits $\beta$-galactosidase $(\beta$ gal) activity within $2 \mathrm{~h}$ of staining. We also performed a quantitative chemiluminescent $\beta$-gal assay in which light emission is measured in a luminometer. However, we were unable to identify highly expressing receptor cell lines, probably due to high background from endogenous enzymatic activities pre- sent in the $\beta$-gal system. Therefore, we could not completely exclude leakage and low induction of gene expression. Moreover, the $\beta$-gal assay is not a rapid screening system because of its long reaction time of up to $60 \mathrm{~min}$. Thus, we generated a firefly reporter gene assay method to rapidly identify highly expressing ecdysone and retinoid $\mathrm{X}$ receptors cell lines. A luciferase reporter system has low background to signal because no endogenous bioluminescence is present. The reaction time was reduced to $15 \mathrm{~s}$. In addition, the reporter performance can be normalized to the transfection efficiency using a control reporter that is not available for chemiluminescent $\beta$-gal assay systems. Using a firefly reporter gene assay system, we successfully established two colon cancer cell lines that stably express receptors for more than six months of continuous culture. In this paper, we elucidated their inducibility and other characteristics.

\section{MATERIALS AND METHODS}

\section{Plasmid Construction}

The pGL2/Enhancer and pGL3/Basic firefly reporter gene plasmids, herpes simplex virus thymidine kinase (TK) promoter-driven Renilla luciferase plasmid pRL/TK and CMV promoterdriven GAL4 cDNA coding plasmid pBIND, were purchased from Promega (Madison, WI, USA). The ecdysone receptor coding plasmid pVgRXR and expression vector pIND plasmid were purchased from Invitrogen. To generate the ecdysone-inducible firefly luciferase reporter genes $\mathrm{pGL} 2 / \mathrm{EcR}$ and pGL3/EcR, the ecdysone receptor re- 
Table 1. Initial Screening of Ecdysone Receptor-expressing cells: pGL2/EcR Reporter Gene Induction

\begin{tabular}{|c|c|c|c|}
\hline \multicolumn{2}{|c|}{ SW480/VgRXR Cells } & \multicolumn{2}{|c|}{ HCT116/VgRXR Cells } \\
\hline Cell Line & Fold Induction & Cell Line & Fold Induction \\
\hline 2 & 6.8 & $\underline{1}$ & $\underline{22.8}$ \\
\hline$\underline{3}$ & $\underline{69.9}$ & 6 & 12.8 \\
\hline$\underline{6}$ & $\underline{81.6}$ & 10 & 15.0 \\
\hline 13 & 16.4 & 18 & 11.4 \\
\hline 14 & 3.6 & $\underline{21}$ & $\underline{54.6}$ \\
\hline 15 & 3.6 & $\underline{24}$ & $\underline{21.8}$ \\
\hline 16 & 10.6 & 27 & 10.9 \\
\hline$\underline{20}$ & $\underline{88.6}$ & 28 & 10.6 \\
\hline$\underline{24}$ & $\underline{85.9}$ & $\underline{31}$ & $\underline{25.0}$ \\
\hline 26 & 3.8 & 37 & 12.0 \\
\hline 27 & 3.5 & $\underline{38}$ & $\underline{27.1}$ \\
\hline 32 & 3.1 & 41 & 6.6 \\
\hline 38 & 3.4 & & \\
\hline 44 & 2.2 & & \\
\hline
\end{tabular}

Highly expressing lines are underlined.

sponsive element was excised from the pIND plasmid using restriction enzymes BglII and HindIII (New England Biolabs, Beverly, MA, USA). Subsequently, the fragment was subcloned into the BglII/HindIII sites of the pGL2/Enhancer or pGL3/Basic plasmids. For the pIND/Luc plasmid construction, the firefly luciferase cDNA was excised from the pGL3/Basic plasmid using BglII and XbaI and subcloned into the $\mathrm{BamHI} / \mathrm{XbaI}$ sites of the ecdysone expression vector pIND plasmid. EGFP cDNA was excised from the pp53EGFP plasmid (Clontech Laboratories, Palo Alto, CA, USA). Subsequently, pIND/EGFP was generated by subcloning the cDNA fragment into the $B a m \mathrm{HI} / X b a \mathrm{I}$ sites of the pIND plasmid.

\section{Establishment of SW480/VgRXR and HCT116/VgRXR Cell Lines}

SW480 and HCT116 human colon cancer cell lines were obtained from the American Type Culture Collection (Manassas, VA, USA). SW480 cells were cultured in phenol red-free Leibovitz's L-15 medium supplemented with $10 \%$ heat-inactivated FBS (both from Life Technologies, Rockville, $\mathrm{MD}, \mathrm{USA}$ ) in a $0.8 \% \mathrm{CO}_{2}$ incubator at $37^{\circ} \mathrm{C}$. HCT 116 cells were maintained in phenol red-free RPMI 1640 medium (Life Technologies) supplemented with $10 \% \mathrm{FBS}$ in a $5 \% \mathrm{CO}_{2}$ incubator at $37^{\circ} \mathrm{C}$. One microgram of $\mathrm{pVgRXR}$ (Invitrogen) plasmid encoding the ecdysone receptor was transfected into $1 \times$ $10^{5}$ SW480 or HCT116 cells, respectively, using FuGENE ${ }^{\mathrm{TM}} 6$ (Roche Molecular Biochemicals, Indianapolis, IN, USA) according to the manufacturer's protocol. After $24 \mathrm{~h}$ incubation, $1 \mathrm{mg} /$ $\mathrm{mL}$ Zeocin (Invitrogen) was added to the medium and cultured for an additional two weeks. Individual colonies were trypsinized, seeded, and expanded. A sample of each clone was transfected with pGL2/EcR and cultured for $48 \mathrm{~h}$ in the presence or absence of 10 $\mu \mathrm{M}$ Ponasterone A (Invitrogen). In total, $50 \mathrm{SW} 480 / \mathrm{VgRXR}$ and HCT116/ VgRXR clones were picked, and their inducibility was measured using the Dual-Luciferase Reporter Assay System (Promega). The clones demonstrating the highest inducibility were used for further studies.

\section{Inducibility of SW480/VgRXR and HCT116/VgRXR Cells}

Cells $\left(2.5 \times 10^{4}\right)$ were seeded into each well of a 24 -well plate. After $24 \mathrm{~h}$, cells were co-transfected with $200 \mathrm{ng}$ 
each inducible reporter gene plasmid and $50 \mathrm{ng}$ TK promoter-driven Renilla luciferase reference plasmid (pRL/TK) using the TransFast ${ }^{\mathrm{TM}}$ lipofection kit (Promega) according to manufacturer's recommended protocol. All transfections were performed in triplicate. Cultured for $24 \mathrm{~h}$ in the presence of various concentrations of Ponasterone A or dimethyl sulfoxide (DMSO). Cells were then lysed with $100 \mu \mathrm{L}$ lysis buffer (Dual-Luciferase Reporter Assay System), and $30 \mu \mathrm{L}$ lysate were assayed for the luciferase activity using Micro Lumat Plus ${ }^{\mathrm{TM}}$ LB96V (Wallac/ Belthold, Wellesley, MA, USA).

\section{Immunoblotting}

Cells $\left(2 \times 10^{5}\right)$ were seeded into the 6-well dishes and cultured for $24 \mathrm{~h}$. Subsequently, cells were transfected with $1 \mu \mathrm{g}$ pIND/EGFP and cultured in the presence or absence of Ponasterone A for $24 \mathrm{~h}$. CMV promoter-driven GAL4 cDNA coding plasmid (pBIND) $(0.5 \mu \mathrm{g})$ was co-transfected in each sample as an internal control for transfection efficiency. Cells were washed twice with PBS and lysed in $200 \mu \mathrm{L}$ SDS-PAGE sample buffer (Bio-Rad Laboratories, Hercules, CA, USA). Cell lysate samples $(10 \mu \mathrm{L})$ were separated on a $4 \%-20 \%$ acrylamide linear gradient SDS-PAGE gel (Bio-Rad Laboratories), transferred onto nitrocellulose membrane, and blotted with an antiGFP and anti-GAL4 antibody (Santa Cruz Biotechnology, Santa Cruz, CA, USA). Signals were visualized using the ECL ${ }^{\circledR}$ kit (Amersham Pharmacia Biotech, Little Chalfont, UK).

\section{RESULTS}

After selection with Zeocin for two weeks, 50 independent colonies from heterodimeric ecdysone receptor-transfected SW480 and HCT116 cells were screened with our pGL2/ EcR reporter plasmid and the Dual-Luciferase Reporter Gene Assay (Table 1). Four and five clones highly expressing the ecdysone receptors were obtained from SW480 and HCT116, respectively (SW480/VgRXR and HCT116/ VgRXR). The highest inducible clones from SW480/VgRXR and HCT116/
VgRXR showed about 90- and 55-fold induction, respectively, after $24 \mathrm{~h}$ treatment with $10 \mu \mathrm{M}$ Ponasterone A. We continuously cultured the cells (SW480/ VgRXR no. 20 and HCT116/VgRXR no. 21) to ascertain stable expression.
After six months, these cells maintained high inducibility (Figure 1, A, B, C, and $D)$. However, the magnitude of the inducibility was reduced. SW480/VgRXR and HCT116/VgRXR cells showed about 20- and 10-fold induction, respec-
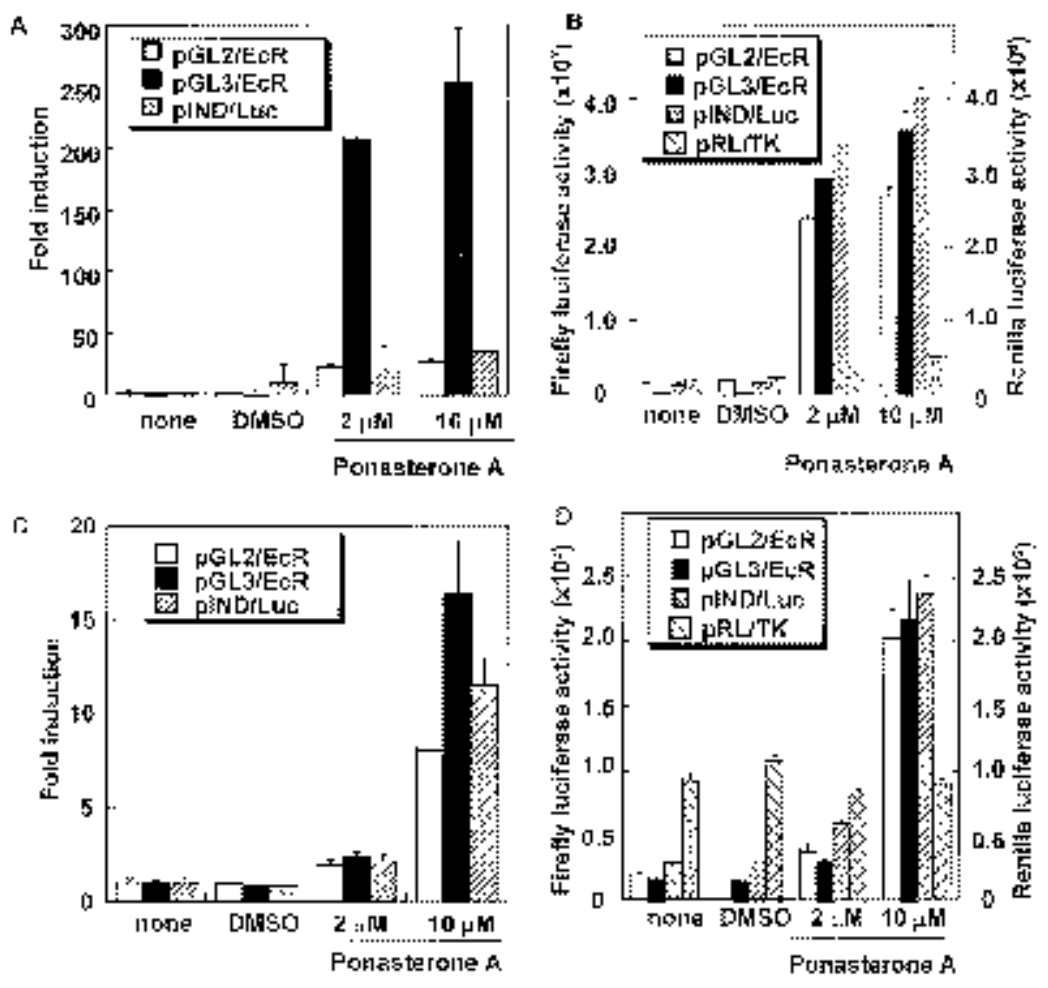

E

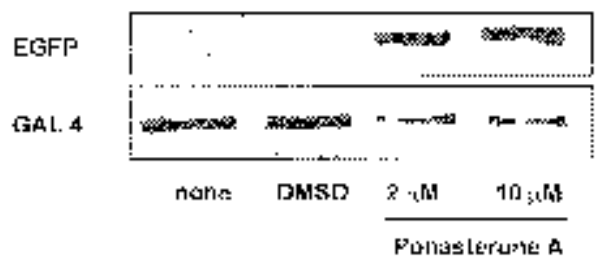

Figure 1. Reporter gene induction in ecdysone receptor-expressing SW480/VgRXR cells and HCT116/VgRXR cells. Cells were transfected with pGL2/EcR, pGL3/EcR, or pIND/Luc and cultured for $24 \mathrm{~h}$ in the presence or absence of Ponasterone A. TK promoter-driven Renilla luciferase plasmid (pRL/TK) was co-transfected in each sample as an internal control for transfection efficiency. The ecdysone receptor responsive element from the pIND ecdysone expression vector was subcloned into either pGL2/Enhancer or pGL3/Basic firefly luciferase reporter plasmid (pGL2/EcR or pGL3/EcR). Luciferase cDNA was subcloned into the pIND expression vector (pIND/Luc). (A and B) SW480/VgRXR cells. (C and D) HCT116/VgRXR cells. (A and C) Comparison of the inducibility. Cells were transfected with pGL2/EcR, pGL3/EcR, or pIND/Luc, respectively. Firefly luciferase activity was normalized against Renilla luciferase activity in SW480/VgRXR cells. (B and D) Induction of firefly luciferase activity by pGL2/EcR, pGL3/EcR, or pIND/Luc and reference Renilla luciferase activity by pRL/TK. (E) Immunoblot analysis of EGFP induction in SW480 cells. Cells were transfected with $1 \mu \mathrm{g}$ pIND/EGFP and cultured in the presence or absence of Ponasterone A for $24 \mathrm{~h}$ before immunoblotting. CMV promoter-driven GAL4 cDNA coding plasmid (pBIND) $(0.5 \mu \mathrm{g})$ was co-transfected in each sample as an internal control for transfection efficiency. 
tively, as measured by the same pGL2/ EcR reporter gene after treatment with $10 \mu \mathrm{M}$ ecdysone analogue Ponasterone A for $24 \mathrm{~h}$ (Figure 1, A and C).

In addition, we tested three different kinds of backbone vectors that drove the same luciferase gene. SW480/ VgRXR transfected with either pGL2/EcR or pIND/Luc reporter plasmids showed a more than 20 -fold induction after treatment with $2 \mu \mathrm{M}$ Ponasterone A, whereas HCT116/

A
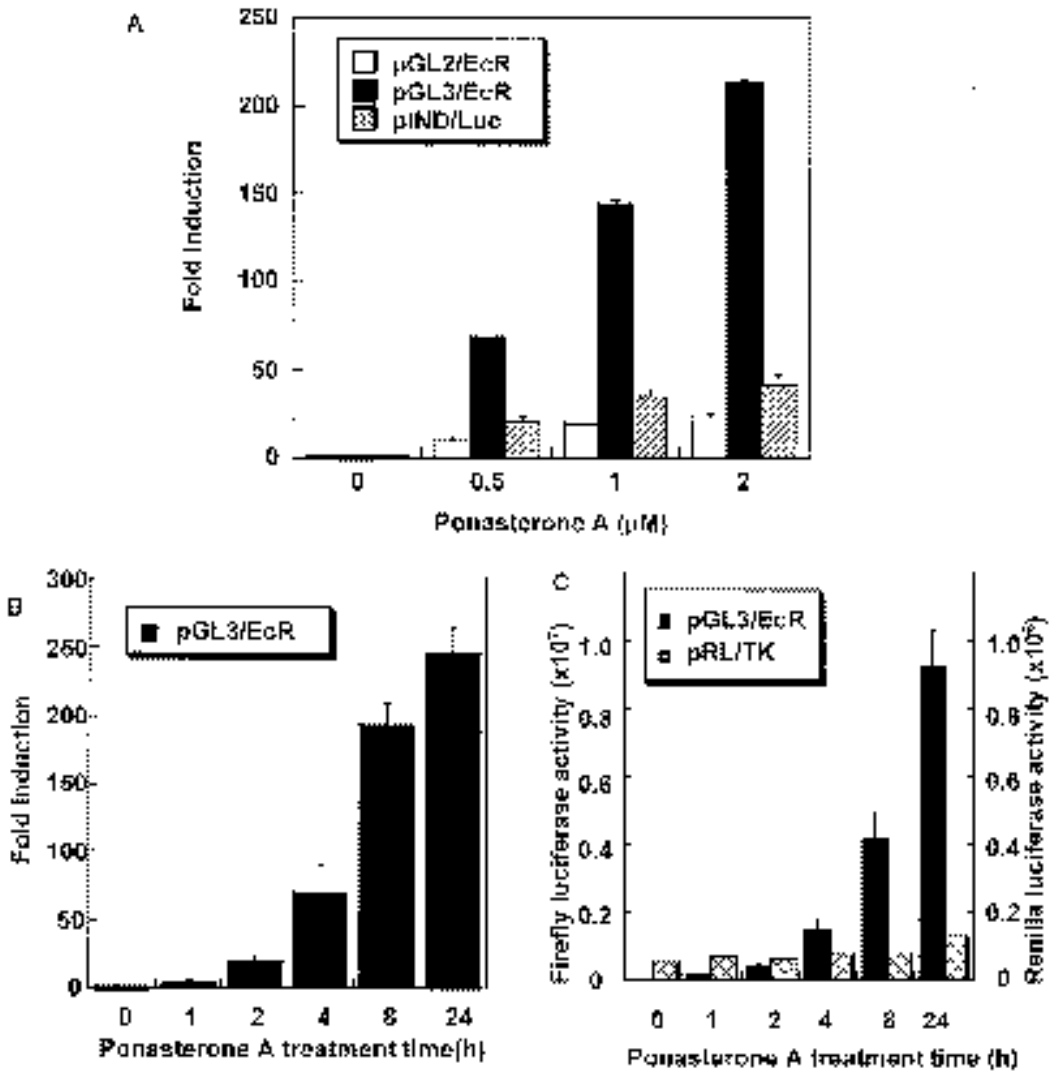

D

EGPP

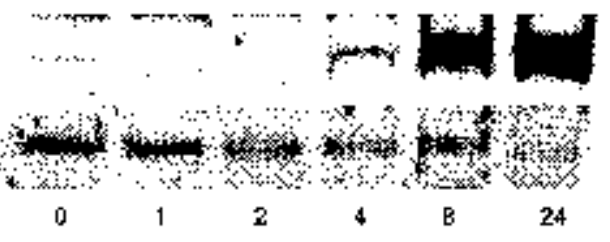

Ponasterone A treatment time (h)

Figure 2. Prompt and efficient response of SW480/VgRXR cells to Ponasterone A. (A) SW480/VgRXR cells treated with Ponasterone A responded in a dose-dependent manner. Cells were cotransfected with the inducible plasmid and the reference plasmid pRL/TK and cultured for $24 \mathrm{~h}$ in the presence or absence of Ponasterone A. Firefly luciferase activity by inducible plasmids was normalized against Renilla luciferase activity in SW480/VgRXR cells. (B, C, and D) Time course of induction. Cells were co-transfected with the pGL3/EcR and pRL/TK plasmids in the presence of $2 \mu \mathrm{M}$ Ponasterone A and collected at the indicated time points up to $24 \mathrm{~h}$. (B) pGL3/EcR plasmid-driven firefly luciferase activity normalized against pRL/TK-driven Renilla luciferase activity. (C) Comparison of inducible firefly luciferase activity and reference Renilla luciferase activity. (D) Immunoblot analysis of EGFP induction in SW480 cells. Cells were transfected with $1 \mu \mathrm{g}$ pIND/EGFP cultured in the presence or absence of Ponasterone A for $24 \mathrm{~h}$ before immunoblotting. CMV promoter-driven GAL4 cDNA coding plasmid (pBIND) $(0.5 \mu \mathrm{g})$ was co-transfected in each sample as an internal control for transfection efficiency. 
VgRXR cells only showed 2-2.5-fold induction. According to these results, SW480/VgRXR cells seemed to have a 10 times better response to the same amount of Ponasterone A. However, the third reporter plasmid used in this assay, pGL3/EcR, demonstrated higher inducibility (Figure 1, A and $\mathrm{C}$, and Figure 2A). Especially in SW480/Vg RXR cells, the better inducibility by pGL3/EcR appeared even at lower concentrations than $2 \mu \mathrm{M}$ Ponasterone A. The induction level of SW480/VgRXR by $\mathrm{pGL} 3 / \mathrm{EcR}$ reporter was about 100 times higher than that of HCT116/ VgRXR cells. There was a significant difference in the inducibility of pGL3/ EcR compared to the other reporter constructs in SW480/VgRXR cells (Figure 1, A and B). Actual firefly luciferase activity measurements indicated that the difference came from the low background of pGL3/EcR in SW480/VgRXR cells (Figure 1, B and D). The level of inducibility reached a plateau at $2 \mu \mathrm{M}$ Ponasterone $\mathrm{A}$ for all three vectors. A similar result was observed when the pIND/EGFP plasmid was used on SW480/VgRXR, and EGFP expression was measured by im munoblot (Figure 1E). EGFP was induced by the addition of $2 \mu \mathrm{M}$ Ponasterone A but did not show a further significant increase at $10 \mu \mathrm{M}$. Reference Renilla luciferase gene expression from $\mathrm{pRL} / \mathrm{TK}$ was not affected by the Ponasterone A treatment up to $10 \mu \mathrm{M}$ (Figure 1, B and D).

We tested the response of SW480/ VgRXR cells to lower concentrations of Ponasterone A than $2 \mu \mathrm{M}$ to find optimum conditions (Figure 2A). Within the range between 0.5 and $2 \mu \mathrm{M}$ Ponasterone A treatment, luciferase activity was increased in a dose-dependent manner. This suggests that the tight regulation of the transgene in SW480/VgRXR cells by Ponasterone $A$ is in the range of $1-2 \mu \mathrm{M}$. Next, a time course study was performed using $2 \mu \mathrm{M}$ Ponasterone A (Figure 2B). Gene induction was already observed after 2 $\mathrm{h}$ and reached 200-fold induction after $8 \mathrm{~h}$ (Figure 2, B and C). The induced expression of firefly luciferase continued up to $24 \mathrm{~h}$. On the other hand, the Renilla luciferase activity driven by TK promoter indicated that the transfection efficiency was equal. This observation was confirmed with protein blot using the pIND/EGFP plasmid onto SW480/ VgRXR cells (Figure 2D). The target gene expression was indeed induced promptly and efficiently.

\section{DISCUSSION}

Conditional expression systems for proteins in mammalian cells are potent tools for studying the function of a gene and its corresponding protein. Those systems do not require physiological stimulation to activate the target gene. Therefore, the function of the protein can be analyzed without influence of upstream signaling pathways. Establishment of a cell line that allows induction of the target gene expression using these systems makes it possible to study the target gene functions under highly reproducible conditions. Tight regulation of gene expression system makes it possible to induce high levels of the cell death proteins (1) or cell growth-inhibitory molecules (6).

There are several widely used conditional expression systems for mammalian cell lines, in particular the tetracycline-inducible promoter (2), the metallothioneine promoter (5), the Lac repressor system (3), and the ecdysoneinducible system (4). The choice of the system is determined by the sensitivity of the cell lines towards the inducers used. For this reason, the ecdysone-inducible system may not be optimal for hormone-sensitive cell types, such as breast cancer cell lines. This system shows high sensitivity towards the addition of steroid hormones. Except for the metallothioneine promoter system, all the systems consist of two or more components supplied by different plasmid vectors to allow the expression of the target gene by either a repressionor an activation-based method.

From our study, we conclude that it is advantageous to first generate a stable cell line expressing high levels of functional modulator protein. Our new technique based on a widely used luciferase reporter gene assay allows the rapid identification of highly inducible receptor-expressing cells. Furthermore, this method is useful to determine the dynamic range of induction using various concentrations of inducers.

\section{ACKNOWLEDGMENTS}

The authors gratefully acknowledge Drs. Mike Fried, Stefan Ries, Carola Biederer, Christian Brandts, and Masashi Aonuma for suggestions and critical reading of this manuscript. We thank members of McCormick and Stokoe Labs for their helpful discussion. O.T. is a recipient of a Research Fellowship from Uehara Memorial Foundation, Tokyo, Japan in 2000. This study was supported by a grant from Daiichi Pharmaceutical Co. Ltd., Tokyo, Japan, for Daiichi Cancer Research Program at University of California, San Francisco.

\section{REFERENCES}

1.Gil, J., H. Yamamoto, J.M. Zapata, J.C. Reed, and M. Perucho. 1999. Impairment of the proapoptotic activity of Bax by missense mutations found in gastrointestinal cancers. Cancer Res. 59:2034-2037.

2.Gossen, M. and H. Bujard. 1992. Tight control of gene expression in mammalian cells by tetracycline-responsive promoters. Proc. Natl. Acad. Sci. USA 89:5547-5551.

3.Hu, M.C. and N. Davidson. 1987. The inducible lac operator-repressor system is functional in mammalian cells. Cell 48:555-566.

4.No, D., T.P. Yao, and R.M. Evans. 1996. Ecdysone-inducible gene expression in mam malian cells and transgenic mice. Proc. Natl. Acad. Sci. USA 93:3346-3351.

5.Searle, P.F., G.W. Stuart, and R.D. Palmiter. 1985. Building a metal-responsive promoter with synthetic regulatory elements. Mol. Cell. Biol. 5:1480-1489.

6.Wang, Y., G. Blandino, M. Oren, and D. Givol. 1998. Induced p53 expression in lung cancer cell line promotes cell senescence and differentially modifies the cytotoxicity of anticancer drugs. Oncogene 17:1923-1930.

Received 31 October 2001; accepted 15 March 2001.

Address correspondence to:

Dr. Osamu Tetsu

University of California, San Francisco

School of Medicine

Comprehensive Cancer Center

Cancer Research Institute

San Francisco, CA 94143-0128, USA

e-mail: otetsu@cc.ucsf.edu

For reprints of this or any other article, contact Reprints@BioTechniques.com 